\title{
Intrapartum Fetal Stimulation
}

National Cancer Institute

\section{Source}

National Cancer Institute. Intrapartum Fetal Stimulation. NCI Thesaurus. Code C114084.

Any method used to elicit a fetal heart rate acceleration response during labor. 